\title{
Aproximación a la representación fraseográfica del lema de las locuciones*
}

MOHAMMED BOUGHABA **

Recepción: 06 de enero de 2019

Aprobación: 13 de junio de 2019

Forma de citar este artículo: Boughaba, M. (2019). Aproximación a la representación fraseográfica del lema de las locuciones. Cuadernos de Lingüística Hispánica, (34). pp 41-66.

(6i) $10.19053 / 0121053 X . n 34.2019 .9595$

* Artículo de reflexión.

** Doctor en fraseología y fraseografía, Universidad Hassan II de Casablanca, Marruecos. Profesor de español como lengua extranjera. Correo electrónico: boughaba.md@gmail.com . 다ttps://orcid.org/0000-0003-3653-1287 


\section{Resumen}

El establecimiento del lema de las locuciones suscita una serie de escollos. Dichos escollos se agudizan más, porque el lema, en este caso, no corresponde a un lexema simple, sino a una unidad pluriverbal. En efecto, muchas veces resulta complejo decidir bajo qué componente de los que constituyen la locución debe ser lematizada, lo que explica por qué los diccionarios no están de acuerdo a la hora de determinar el lema de este tipo de unidades fraseológicas (UFS). La presente contribución trata de mostrar cómo es descrito el lema de las locuciones en varios diccionarios monolingües generales y fraseológicos. Para ello, vamos a esclarecer varias cuestiones relacionadas con este tema, como la forma canónica, los elementos del contorno, la inserción de algunos verbos en el lema, el régimen preposicional, etc.

Palabras clave: lema, locución, unidad fraseológica, diccionario.

\section{Approach to Phraseographical Representation of the Lemma of Locutions}

\section{Abstract}

Setting of locutions' lemma raises a number of pitfalls. These pitfalls become more acute, because the lemma, in this case, does not correspond to a simple lexeme, but a multiverbal unit. In fact, it is often difficult to decide under which component of those that make up the locution should be lemmated, which explains why dictionaries do not agree when determining the lemma of this type of phraseological units. This contribution tries to show how the lemma of locutions is described in several general monolingual and phraseological dictionaries. To do this, we will clarify several issues related to this topic, such as the canonical form, the contour elements, the insertion of some elements in the lemma, the prepositional regime, etc.

Keywords: lemma, locution, phraseological unit, dictionary.

\section{Aproximación a la representación fraseográfica del lema de las locuciones}

Rapprochement à la représentation phrase-graphique de la devise des locutions

\section{Résumé}

Établir la devise des locutions suscite une série d'écueils. Ceux-ci deviennent particulièrement aigus, parce que la devise, dans ce cas, ne correspond pas à un lexème simple, mais à une unité pluri-verbale. En effet, il est souvent complexe de décider, parmi tous les composants qui constituent la locution, sous lequel cette unité-là doit être clas- 
sée. Ceci explique pourquoi les dictionnaires ne se mettent pas d'accord au moment de déterminer la devise de ce type d'unités phraséologiques (UFS). Ce travail essaie de montrer comment on décrit la devise des locutions dans plusieurs dictionnaires monolingues généraux et phraséologiques. Pour y arriver, on va éclaircir plusieurs questions liées avec ce sujet, comme la forme canonique, les éléments de l'aperçu, l'insertion de quelques verbes dans la devise, le régime prépositionnel, etc.

Mots clés: devise, locution, unité phraséologique, dictionnaire.

Aproximação da representação fraseográfica do lema de locuções

\section{Resumo}

0 estabelecimento do lema de locuções levanta uma série de armadilhas. Essas armadilhas se tornam mais agudas, porque o lema, neste caso, não corresponde a um simples léxico, mas a uma unidade multiverbal. De fato, muitas vezes é difícil decidir sob qual componente daqueles que compõem a frase deve ser lemado, o que explica por que os dicionários não concordam ao determinar o lema desse tipo de unidades fraseológicas (UFS). A presente contribuição tenta mostrar como a frase das locuções é descrita em vários dicionários monolíngues e fraseológicos gerais. Para isso, esclareceremos várias questões relacionadas a esse tópico, como a forma canônica, os elementos de contorno, a inserção de alguns verbos no lema, o regime preposicional etc.

Palavras-chave: slogan, frase, unidade fraseológica, dicionário.

\section{Introducción}

En todo artículo lexicográfico hay que distinguir entre el lema y el cuerpo del artículo. El lema o locución-guía (según la terminología usada por Torrent-Lenzen, 2007:283) es la forma que se adopta como representante de todas las demás formas flexionadas de una locución, la cual encabeza cada uno de los artículos del diccionario. El establecimiento del lema de las locuciones, al igual que otros aspectos de la práctica lexicográfica, suscita una serie de escollos. Tratándose de unidades pluriverbales, dichos escollos se agudizan más, porque el lema, en este caso, no corresponde a una palabra, sino a una combinación de palabras. Esta razón, es decir el carácter pluriverbal de las UFS, explica por qué las locuciones no se registran como lemas en los diccionarios generales, sino como sublemas, pues el principio que rige el establecimiento de los lemas en la tradición lexicográfica se basa en los lexemas simples y no en las combinaciones de palabras. Además, y como sostiene Werner (1982: 218), "el concepto de 'lema' no se 
funda en una definición creada por la lingüística moderna, sino en una tradición plurisecular de la lexicografía".

Antes de tratar los escollos de la delimitación del lema, conviene señalar que la forma canónica de los lexemas simples, así como de las unidades plurilexemáticas, se representa tipográficamente en negrita y en minúscula, como en cruzarse de brazos $\left(\right.$ Clave $\left.^{I}\right)$, hacer una escapada $\left(D F D E A^{2}\right)$, de buena boca $\left(D L E^{3}\right)$, a carta cabal $\left(D F E M^{4}\right)$, echarse a los pies $\left(D I C L O C V E R^{5}\right)$, etc.

Ahora bien, la dificultad del establecimiento del lema de una unidad fraseológica (UF) empieza desde el momento en que se plantea decidir su forma canónica o básica. Muchas veces no es asequible determinar esta forma básica, debido a la complejidad formal de las UFS y las posibles variaciones en su estructura, sin olvidar las distintas modificaciones y manipulaciones creativas que puedan sufrir. Estos escollos han dado lugar a la aparición de distintos procedimientos en la presentación de las locuciones en los artículos lexicográficos. De hecho, no todas las locuciones son presentadas de la misma manera en los diccionarios. Antes de comentar algunos ejemplos concretos, queremos arrojar luz sobre las fuentes que se emplean para determinar la forma canónica de una UF.

De acuerdo con Penadés Martínez (cfr. 2015: 113), el establecimiento del lema se somete a dos principios: uno teórico y otro práctico. Según la perspectiva teórica, la fijación del lema de una locución tiene que realizarse siguiendo las pautas trazadas por la tradición lexicográfica, la cual trata las UFS como lexemas simples. De este modo, los lemas deben ser recogidos tal y como figuran en los diccionarios generales. Es común proceder de esta forma, puesto que existe una tendencia a considerar como formas canónicas aquellas que aparecen en los diccionarios más difundidos y representativos. Por otra parte, y según la perspectiva práctica, el establecimiento del lema debe tomar en consideración el uso que hacen los hablantes de las locuciones estudiadas. Para ello, se debe optar por otras fuentes de consulta, como los corpora electrónicos de la RAE y los motores de búsqueda en la red, los cuales sirven para dar cuenta del uso real y la frecuencia

1 Clave. Diccionario de uso del español actual.

2 Diccionario fraseológico documentado del español actual. Locuciones y modismos españoles.

3 Diccionario de la Lengua Española.

4 Diccionario fraseológico del español moderno.

5 Diccionario de locuciones verbales para la enseñanza del español. 
de uso de cada unidad. Proceder según este principio práctico puede implicar que los resultados obtenidos sean distintos de los ofrecidos por los diccionarios generales.

La consulta tanto de diccionarios como de otros recursos puede, según Olímpio de Oliveira Silva, "dar un mínimo de garantía" a la hora de determinar la forma canónica:

Sólo una comparación exhaustiva entre las muestras sacadas de las diversas fuentes lexicográficas y el uso conjunto de los múltiples recursos de selección podrán dar un mínimo de garantía a la hora de establecer la forma de la UF que se registrará en el diccionario. (Olímpio de Oliveira Silva, 2004: 195).

Tras el estudio de la forma básica de varios diccionarios, hemos constatado que los resultados no son coincidentes para todos los lexicógrafos, y el principio más adoptado es el teórico, es decir aquel que se basa en calcar las mismas formas canónicas que aparecen en otras compilaciones. En este aspecto, Penadés Martínez (2015: 113) sugiere que "el uso lingüístico debe prevalecer sobre la tradición lexicográfica de reproducir un lema tal y como habitualmente se ofrece en las obras lexicográficas precedentes". En lo que sigue se tratan algunos aspectos que reflejan la heterogeneidad en el establecimiento de los lemas de las locuciones.

\section{La inserción de algunos verbos en el lema}

Uno de los problemas que se plantean a la hora de establecer la forma canónica de una locución es verificar los elementos constitutivos que son propios de ella. Así, por ejemplo, existe la duda de considerar los siguientes verbos como elementos integrantes en sus respectivas locuciones o no:

- ponerse a bien

- defender a capa y espada

- esperar como agua de mayo

- coger con las manos en la masa

- mandar a freír espárragos

Si tomamos como ejemplo la locución a capa y espada, notamos que aparece registrada con el verbo defender en el DLE (defender a capa y espada a alguien o algo), mientras que tanto el DFDEA como el Clave optan por un lema en el que se descarta ese verbo (a capa y espada). El DFDEA indica explícitamente, en el cuerpo del artículo, que es una locución que se usa "normalmente con el verbo defender", mientras que $e l$ Clave lo hace implícitamente mediante el ejemplo de uso: "defenderá su postura a capa y espada". La decisión de incluir o no el verbo defender en el lema ha tenido repercusiones 
sobre otros aspectos, como la clase de la locución: mientras el $D L E$ considera que es una locución verbal, tanto el DFDEA como el Clave indican que es una locución adverbial. La consideración de un verbo como parte constitutiva de una locución o no debe basarse en su frecuencia de uso y de coaparición con los otros elementos de la unidad. Para llegar a tal conclusión, se puede recurrir a los corpora de la RAE, con el fin de averiguar la forma con la que suele aparecer la UF en cuestión. Los ejemplos de uso sacados del CREA demuestran que, efectivamente, la locución a capa y espada aparece muy frecuentemente con el verbo defender, sin embargo se registran varios casos en los que sale con otros verbos, como proteger, pelear y sustantivos como duelo:

-El afortunado sigue escondido y protegido a capa y espada por la comunidad de inmigrantes africanos de la población. (1994, La Vanguardia, El colectivo africano de Calella oculta al ganador de la Primitiva, Barcelona, España, 05. Actualidad).

Y, está duro, pero nos vamos acostumbrando. Este es un grupo que todo lo peleó a capa y espada. Habrá que trabajar más para que lo nuestro le llegue al público de otros países, al nuestro. Es difícil. (2002: Clarín, Entrevista con el bahiano, Buenos Aires, Argentina, 04. Música).

Entre el espionaje comercial y el duelo a capa y espada suele haber un trecho diminuto. Al parecer, el militar francés Bolley empezó sus trabajos con la compra de planes de defensa antes de profundizar en secretos más importantes y delicados. (1985: ABC, La Administración de la Unión India, bajo el síndrome de la "conexión extranjera", Madrid, España, 03. Política).

Por otra parte, se deja notar que la parte invariable de la locución es a capa y es$p a d a$, mientras que el verbo defender puede aparecer en forma de sustantivo (defensor), o permitir la intercalación de otros elementos ajenos. Veamos los siguientes ejemplos:

El pueblo, ese cándido pueblo, lo había elegido como defensor a capa y espada de sus derechos y legítimos intereses. A lo mejor, el pueblo tenía razón, y aquel señor defendería sus derechos y sus intereses. (1975: Palou, Inés, Barcelona, España, Carne apaleada, 07. Novela)

La Campaña Nacional por el Aborto, mientras tanto, ha defendido el derecho de Lynn a capa y espada, al tiempo que la Alianza Escocesa Pro Vida se ha puesto del lado de James. (1997, ABC, Madrid, España, Un padre escocés lucha en los tribunales para que su ex mujer no abort..., 03. Justicia, legislación).

El examen de los ejemplos citados revela que el verbo defender no debe figurar en el lema de la locución, primero porque no es el único que acompaña la locución y 
también porque permite una serie de variaciones. Los ejemplos de uso ratifican, pues, la categoría de la unidad como locución adverbial y no como verbal. Tomando en consideración estas puntualizaciones, creemos conveniente descartar del lema todos los verbos que no forman parte nuclear ni desempeñan una función semántica inherente a la locución que acompañan. Asimismo, una praxis lexicográfica coherente y clarificadora obliga a presentar, además del lema, los verbos con que suele aparecer la unidad. Este hecho ya ha sido señalado por Zuluaga Ospina en 1992:

Un análisis más completo que los ofrecidos por diccionarios y compilaciones tradicionales debe señalar, además del contenido, las propiedades combinatorias de cada locución, o lo que es lo mismo, su contexto verbal inmediato. (Zuluaga 0spina, 1992: 130).

De ahí, nos parece muy acertado el procedimiento del DFDEA a la hora de establecer el lema, primero recoge solo las partes integrantes de la locución y, luego, en el cuerpo del artículo, señala los verbos con los cuales suele emplearse, como el caso del fraseologismo adverbial con las manos en la masa, en la que indica que se usa "normalmente con los verbos coger, sorprender o pillar" (DFDEA, 2004: 613), o la locución adverbial a la muerte, en cuyo artículo señala que se usa con verbos "como estar, poner y tener" (DFDEA, 2004: 669). Proceder de este modo permite no solo seleccionar la forma básica más conveniente, sino también determinar, con precisión, la clase a la que pertenece cada locución. De hecho, y como sostiene Olímpio de Oliveira Silva:

Muchas unidades tradicionalmente consideradas como LV son, a la luz de los ejemplos de uso, locuciones adjetivas (como curado de espanto (s)), adverbiales (en Babia), pronominales (un higo) o preposicionales (a la altura de). (Olímpio de Oliveira Silva, 2004: 205).

Partiendo de estas premisas, podemos afirmar que solo serían locuciones verbales aquellas cuyo verbo forma parte intrínseca de las mismas. Este tipo de unidades pueden ser totalmente fijas como en darse postín, dormirla, matar al mensajero, frotarse las manos,... o admitir variaciones como en ajustar (o arreglar) cuentas, pillarse (o cogerse) los dedos, etc.

\section{El régimen preposicional}

La inserción de las preposiciones en el lema es otro de los temas controvertidos que no cuentan con el acuerdo común de los especialistas en la materia. Mientras algunos autores como Mellado Blanco (cfr. 2004; 2017) defienden la inclusión de los complementos de régimen preposicional en el lema, porque forman parte indispensable de su 
actualización en el discurso, otros como Olímpio de Oliveira Silva (cfr. 2004) y Penadés Martínez (cfr. 2002-2003 y 2015) opinan que los elementos que introducen el complemento de régimen no constituyen partes integrantes del lema, por lo que deben aparecer separados de la forma canónica, en un apartado específico.

En lo que sigue vamos a presentar algunos ejemplos para demostrar dos aspectos:

1). la heterogeneidad en la delimitación del lema de las locuciones, 2) la vacilación a la hora de insertar las preposiciones.

En lo que se refiere al aspecto de la heterogeneidad y la incongruencia en el establecimiento del lema, podemos citar el ejemplo de la locución echar mano, que ha sido presentada de la siguiente manera en los siguientes diccionarios:

$D L E$ : echar una mano a. (loc. verb. Ayudar a la ejecución de algo'.)

Clave: echar una mano o tender $\{1 \mathbf{1} / \mathbf{u n a}\}$ mano (loc.verb.col. 'Ayudar')

DFDEA: echar una mano (o una manita) [a alguien] (v. Ayudar [le]').

La primera observación que podemos hacer, tras examinar los ejemplos presentados, es la falta de un procedimiento unívoco en la delimitación del lema. Aun tratándose de una misma locución, y las definiciones de los tres diccionarios se corresponden en este sentido, pues los tres indican que significa ayudar, las decisiones tomadas para establecer el lema han sido distintas según la perspectiva de cada diccionario. Incluso, y como vamos a comentar a continuación, un mismo diccionario puede proceder de formas diferentes, para tratar el lema de locuciones iguales en su estructura y en la categoría a la que pertenecen.

En el caso del $D L E$, se observa que ha optado por incluir en el lema la preposición $a$ (echar una mano a..). Ello puede dar a entender que es un elemento constitutivo de la forma canónica de la locución, lo cual es erróneo, pues el papel de la preposición consiste, simplemente, en introducir un complemento.

El DFDEA ha procedido de forma un poco diferente, pues, además de incluir la variación de la unidad (o una manita), ha insertado, entre corchetes, un elemento del contorno ([a alguien]).

El Clave ha decidido descartar la preposición a del lema que registra. Tampoco incluye el actante alguien. No obstante, inserta la variante de la locución echar una mano (tender $\{\mathbf{l a / u n a}\}$ mano). 
Por otra parte, existen otros casos en los que podemos observar distintas formas de proceder en el seno de un mismo diccionario. El DFDEA, por ejemplo, a veces informa de la preposición en un apartado entre corchetes junto al lema, como en echar una mano (o una manita) [a alguien], mientras que en otros casos lo hace en otro apartado junto con la definición, como en:

jugar al escondite.v Esconderse [alguien] para que no le encuentren. Frec con un compl $\underline{\mathrm{CON}}$ que expresa la pers respecto a la cual se esconde.

Por su parte, el DLE ha abogado por insertar la preposición a en echar una mano $a$, mientras que en la locución alargar la mano ha optado por no incluirla, aunque en la definición que ofrece deja claro que es una preposición seleccionada por la locución: 'Tender la mano a otro para saludarlo, solicitando la suya'. Al mismo tiempo, en la locución alzar la mano ha optado por incluir la preposición y el actante: alzar la mano a alguien.

Entonces, aunque estamos ante tres locuciones de la misma clase y la misma estructura (echar una mano, alargar la mano, alzar la mano) y todas evocan la misma preposición (a), el DLE ha tratado el lema correspondiente a cada una de forma distinta:

incluye la preposición como elemento del lema (echar una mano a.), 2) descarta la preposición del lema (alargar la mano), 3) la preposición aparece con los elementos del contorno (alzar la mano a alguien). Es más, podemos añadir otro procedimiento en el que la preposición aparece en negrita y el actante en cursiva, como en tomarla con alguien o algo o dárselas alguien de algo, lo cual da a entender que las preposiciones integran la forma básica de sus respectivas locuciones.

La inserción de elementos preposicionales totalmente ajenos a la forma canónica de la locución, en los lemas, se puede explicar por el hecho de que los diccionarios pretenden proporcionar al usuario un recurso que le sirva para reconocer los elementos preposicionales que se usan habitualmente con cada unidad. No obstante, proceder de esta forma va en contra de la naturaleza de las locuciones y en contra de toda práctica lexicográfica, pues al igual que los lemas correspondientes a verbos como incidir, dejar y volver, no incluyen las preposiciones con las que suelen aparecer (en, de y $a$, respectivamente), para el tratamiento del lema de las locuciones se debería proceder de la misma forma, esto es, incluir solo los elementos constitutivos de la forma canónica de cada unidad. 
Por otra parte, actuar de modos tan distintos y dispares dentro de un mismo diccionario, como el $D L E$, pone en evidencia la praxis lexicográfica adoptada por este diccionario para el tratamiento de las locuciones, lo cual podría repercutir negativamente en la elaboración de otros repertorios que toman el $D L E$ como principal referencia.

Ahora bien, ies posible establecer un procedimiento sistemático para delimitar el lema de las locuciones? Si los diccionarios anteriormente comentados revelan su carácter vacilante y asistemático, existen otras obras que han mostrado más regularidad en el procedimiento que siguen. Es el caso del DICLOCVER, diccionario de locuciones verbales en el que la delimitación del lema se ha realizado de forma más clara y sistemática. Veamos los siguientes ejemplos:

soltarse la lengua $v$. [a alguien].

tener unas palabras $v$. [alguien, con alguien].

irse los ojos $v$. [a alguien, detrás de/tras algo/alguien]

A tenor de los ejemplos citados, se desprende que el DICLOCVER no incluye la preposición en el lema ni los elementos del contorno, pero, eso sí, da cuenta de ellos en otra parte informativa, en la que aparece la preposición junto con los elementos del contorno entre corchetes. Además, se hace mención de las otras preposiciones que pueden combinarse con la misma locución, las cuales aparecen separadas por una barra. Este procedimiento deja claro cuál es la forma canónica de una locución resaltándola en negrita, sin ignorar los demás elementos informativos necesarios para su uso adecuado.

\section{Los elementos del contorno}

El DLE define el término contorno como:

Conjunto de los elementos de la definición que informan sobre el contexto habitual del vocablo definido, en oposición a los elementos que informan sobre su contenido. (DLE, 2014).

La definición del DLE distingue entre dos componentes: 1) los que informan sobre el contexto (elementos del contorno), y 2) los que informan sobre el contenido (elementos que representan la forma canónica).

Olímpio de Oliveira Silva ha sido más específica al indicar que los elementos del contorno asignan: 
Por un lado, determinados límites contextuales en el uso del definido, esto es, los elementos léxicos que pueden combinarse con él y, por otra parte, indican sus posibilidades de construcción sintáctica. (Olímpio de Oliveira Silva, 2004: 219-220).

Son, pues, elementos del contorno los componentes que aparecen entre corchetes en los siguientes ejemplos:

mamarse el dedo [alguien].

no tener precio [algo/alguien].

ponerse morado [alguien, de algo].

Conviene indicar que tanto los lexemas simples como las combinaciones pluriverbales presentan iguales contornos en los diccionarios generales y especializados. La diferencia reside en su ubicación en el artículo lexicográfico (cfr. Mellado Blanco, 2017: 125). Efectivamente, como ya veremos más adelante, los contornos en los diccionarios fraseológicos pueden aparecer en distintos sitios del artículo, dependiendo de la concepción de cada diccionarista. De ahí, una de las preguntas fundamentales que se plantean a la hora de tratar este tema es: ¿en qué parte del artículo lexicográfico se deben incluir los elementos del contorno? Antes de contestar esta pregunta, vamos a presentar las distintas opiniones de los especialistas, así como las posturas adoptadas por algunos diccionarios generales y especializados en el tratamiento del contorno de las locuciones.

En la subsección anterior hemos llamado la atención sobre la necesidad de distinguir entre los elementos realmente constitutivos de una UF y los componentes del régimen preposicional que serán actualizados en el discurso. En lo que sigue se va a

Aproximación a la representación fraseográfica del lema de las locuciones

plantear la misma cuestión, pero esta vez entre los elementos del contorno y la forma canónica. Uno de los objetivos de este apartado es revelar el tratamiento desigual que reciben los elementos del contorno en los diccionarios generales y fraseológicos. Serra Sepúlveda apunta en este aspecto:

Todos los diccionarios de español que dicen ocuparse del contorno de los definidos, lo hacen de manera bastante irregular y, en algunos casos, deficiente. Llaman la atención, sobre todo, las divergencias notables entre unos y otros para describir las mismas unidades. (Serra Sepúlveda, 2006: 219).

Si los diccionarios no están de acuerdo en la forma de presentar los elementos del contorno en el artículo lexicográfico, los fraseólogos tampoco coinciden en este sentido: 
El tratamiento del contorno de las locuciones también suscita desacuerdos entre la postura adoptada por los que consideran que los elementos que lo constituyen, propios de la combinatoria sintagmática de la unidad, no deben incluirse en el lema y los lexicógrafos que entienden que forman parte de la estructura de la locución. (Penadés Martínez, 2010: 203).

A continuación se presentarán varias posturas, algunas defienden la inclusión de los actantes en el lema, otras abogan por separarlos.

Martínez Marín (1996: 65) ha sido pionero en emplear el término contorno en la fraseografía teórica española, para referirse a estos elementos que pertenecen al contenido externo "combinatorio y contextual". De este modo, se puede establecer una distinción entre forma canónica y contorno, los cuales no deben ser tratados conjuntamente en el lema, porque, según el mismo autor (cfr. Martínez Marín, 1996), los elementos del contorno no forman parte interna de la unidad, por lo que deben ser diferenciados tipográficamente mediante los paréntesis u otro procedimiento. Otros autores como Santamaría Pérez (2001), Olimpio de Oliveira Silva (2004), Montoro del Arco (2005), Penadés Martínez (2002, 2002-2003, 2015), Castillo Carballo (2015), entre otros, coinciden con el planteamiento adoptado por Martínez Marín (1996), al señalar la necesidad de separar los elementos del contorno de la forma nuclear de la locución. Montoro del Arco alega en este sentido:

[El contorno] forma parte del fraseologismo, pero no de los componentes materiales fijos de este, ya que constituye un hueco funcional que puede ser saturado por elementos que no están fijados sino que son parte, por tanto, de la sintaxis libre, que simplemente están sujetos a las circunstancias contextuales concretas del acto comunicativo. (Montoro del Arco, 2005: 129).

Por su parte, Castillo Carballo sostiene en este sentido:

En muchas ocasiones, en los diccionarios se descuida la correcta distinción del contorno lexicográfico y se tiende a integrarlo como un elemento más de la unidad fraseológica o bien se prescinde de él sin más. (Castillo Carballo, 2015: 51)

Es cierto que la mayoría de los autores han adoptado y apoyado plenamente el procedimiento propuesto por Martínez Marín (1996), es decir, están de acuerdo con la distinción y la separación de los elementos del contorno de la forma básica de las UFS, no obstante, existen otros especialistas que no parecen estar muy convencidos de tal procedimiento. Entre estos autores, destaca Mellado Blanco, quien, en una réplica a la intervención de Martínez Marín sobre el contorno lexicográfico en el I Coloquio Galego 
de Fraseoloxía (Conca i Martínez, 1998: 180-182), ha afirmado que los componentes del contorno- o los actantes o argumentos según la terminología usada por la autora- deben figurar integrados en el lema, porque, de lo contrario, la interpretación del fraseologismo y su uso pueden ser incorrectos por parte de los usuarios, sobre todo si son extranjeros. Es más, califica de "erróneo" el uso de los paréntesis para distinguir los actantes de los demás elementos de la forma canónica, porque, según ella, los paréntesis se usan normalmente para los actantes facultativos y, por tanto, su aplicación a los argumentos obligatorios incidiría más en la confusión. Además, no ve la necesidad de aplicar tal distinción, porque los datos respectivos a los elementos del contorno se dan de antemano en las instrucciones del diccionario, por lo que no supondría ninguna confusión colocar los actantes al mismo nivel que el resto de los elementos que forman el lema de la unidad. Martínez Marín, en respuesta al planteamiento de Mellado Blanco, indica que no se puede proceder con la forma tradicional ubicando los actantes en el mismo nivel que los componentes del lema, porque son dos elementos distintos y deben ser bien diferenciados. De acuerdo con este autor, es la integración de los actantes en el lema lo que puede confundir a los usuarios extranjeros y no lo contrario.

Frente a las dos posturas expuestas, la que apoya la distinción de los elementos del contorno de la forma básica de la unidad y la que defiende la inclusión de los dos elementos en el lema, existe un tercer planteamiento que parece inclinarse más al primer razonamiento, pero lo hace con ciertas precisiones. Se trata del procedimiento seguido por Penadés Martínez (2002-2003, 2008b y 2015), en el que propone, no solo separar los actantes de la forma nuclear, sino que tal separación tiene que aplicarse en un apartado propio.

Para que quede claro, vamos a presentar algunos ejemplos con el planteamiento propuesto por cada uno de los autores citados:

\begin{tabular}{|l|l|l|}
\hline \multicolumn{1}{|c|}{ Mellado Blanco } & \multicolumn{1}{c|}{ Martínez Marín } & \multicolumn{1}{c|}{ Penadés Martínez } \\
\hline $\begin{array}{l}\text {-estar alguien de } \\
\text { broma }\end{array}$ & $\begin{array}{l}\text {-estar (alguien) es } \\
\text { broma }\end{array}$ & $\begin{array}{l}\text {-estar de broma. } \\
\text { [alguien]. }\end{array}$ \\
\hline $\begin{array}{l}\text {-tomar alguien el pelo a } \\
\text { alguien. }\end{array}$ & $\begin{array}{l}\text {-Tomar (alguien) el pelo } \\
\text { (a alguien). }\end{array}$ & $\begin{array}{l}\text {-tomar el pelo. [alguien, } \\
\text { a alguien]. }\end{array}$ \\
\hline $\begin{array}{l}\text {-echar alguien algo } \\
\text { en cara a alguien. }\end{array}$ & $\begin{array}{l}\text {-echar (alguien algo) en } \\
\text { cara (a alguien). }\end{array}$ & $\begin{array}{l}\text {-echar en cara. [alguien, } \\
\text { algo, a alguien ]. }\end{array}$ \\
\hline
\end{tabular}

Tabla 1: La representación de los elementos del contorno según Mellado Blanco, Martínez Marín y Penadés Martínez. 
Son, pues, tres los procedimientos principales que se pueden adoptar a la hora de establecer la estructura de los actantes, cada uno tiene sus propios razonamientos. Penadés Martínez (2002-2003: 117-118) cita cuatro motivos para explicar por qué los actantes deben figurar en un apartado independiente del lema:

- el usuario del diccionario, y sobre todo cuando es un extranjero, puede pensar que los elementos del contorno son constitutivos de la locución.

- la aparición del contorno en el lema de la UF puede distorsionar la presentación de este último por obligar a una concordancia que impide la reproducción de la locución en su forma canónica.

- los elementos del contorno no se incluyen ni se excluyen de manera sistemática en el lema de todas las UFS, ni aun en un mismo diccionario.

- no existe unanimidad de criterios entre los lexicógrafos acerca de cuáles y cuántas sean las unidades que permitan reproducir los elementos del contorno de una unidad fraseológica.

Además, la autora (cfr. Penadés Martínez, 2015: 121) señala que para establecer el lema de las locuciones se debe proceder de la misma forma que adoptan los diccionarios generales a la hora de establecer el lema de las unidades simples. Así, por ejemplo, al igual que un verbo como llenar excluye los actante que exige (alguien llena algo/alguien, de o con algo) y aparece registrado con la forma infinitiva llenar, la locución llenarse la boca, no tiene por qué incluir sus actantes a alguien se le llena la boca con algo

Por otra parte, la autora (cfr. Penadés Martínez, 2015: 119) señala una serie de locuciones recogidas en el DFEM para demostrar que la inclusión de los actantes en el lema puede tergiversar la verdadera forma canónica de las locuciones:

\section{[ser u.p.] cerrada de mollera.}

[ser u.p.] echada para adelante.

[ser/estar u.p.] entrada en carnes.

\section{[estar/ser u.p.] tocada del ala.}

De acuerdo con la praxis lexicográfica, las locuciones que admiten variación de género y/o número tienen que figurar en masculino singular, por lo que la forma básica de las unidades presentadas tiene que aparecer así: cerrado de mollera, echado para 
adelante, entrado en carnes y tocado del ala. Sin embargo, y como el DFEM ha incluido el actante u.p. (una persona) en el lema, ha tenido que alterar la forma canónica para que concuerde con el género femenino del actante. Dicho procedimiento es, según la autora (Penadés Martínez, 2015: 120) "contrario a toda práctica lexicográfica", porque "las formas no marcadas: el masculino y el singular, son las que se reflejan en la forma canónica de las unidades lingüísticas que muestran estas variaciones morfológicas".

Otro aspecto de la disparidad del tratamiento del contorno es el hecho de presentar los actantes de distintas formas. Así, mientras en algunos diccionarios aparecen situados en el mismo nivel que los elementos del contorno, en otros figuran distinguidos con algún procedimiento: entre paréntesis, corchetes o en otra letra. Citemos la locución dar un repaso para ilustrar tal disparidad:

dar un repaso a alguien. (DLE)

dar un rapaso [a algo]/ dar un repaso. [a alguien] (DFDEA).

dar u.p. un (buen) rapaso a alguien/algo. (DFEM)

dar un repaso. (Clave)

La incongruencia y la falta de unanimidad caracterizan, pues, el tratamiento que reciben los elementos del contorno en los diccionarios estudiados. Así, en el DLE la forma básica aparece en negrita, mientras que el actante aparece distinguido con una tipografía normal. El DFDEA hace lo mismo, es decir, distingue la base canónica de los actantes, pero estos, a diferencia del DLE, aparecen entre corchetes. Además, el DFDEA ofrece dos acepciones para esta locución, una con el actante alguien y otra con algo. En el DFEM figuran tres actantes, u.p., alguien y algo, los cuales aparecen en negrita al igual que el resto del lema y sin distinción tipográfica. El Clave, sin embargo, no hace referencia a ningún actante.

Es más, esta falta de sistematicidad se presenta, incluso, en el seno de la misma obra. De hecho, son varios los diccionarios en los que los elementos del contorno han recibido un tratamiento desigual en su microestructura. Uno de los aspectos más llamativos en este sentido, es el hecho de indicar los actantes en algunas entradas, y descartarlos en otras, como el caso del Clave que no señala el argumento en el ejemplo anterior, pero sí lo indica en muchos otros casos, como echar en falta algo, en manos de alguien, estar algo en la mano de alguien, etc. El DFDEA, por su parte, tampoco tiene una concepción regular en este aspecto. Obsérvense los siguientes casos: 
- no registra el actante en el lema, pero da cuenta de él en la definición: morderse la lengua. $\mathrm{v}$ (col) Abstenerse [alguien] a decir lo que quisiera.

- no registra el actante en el lema ni da cuenta de él en la definición: tocar madera. $\mathrm{v}$ (col) Realizar el gesto físico de tocar un objeto de madera para conjurar una desgracia 0 un peligro.

- registra un actante y discrimina otro: arrastrase a los pies [de alguien]. v Impedir [le] actuar. Los dos argumentos deberían aparecer: arrastrase \alguien] a los pies [de alguien].

— registra los actantes, cada uno en su sitio: meterse [una pers.] en la piel $(o$, más raro, dentro de la piel) [de otra]. v Ponerse en [sus] circunstancias.

— registra todos los actantes al final del lema: dar fatiga [algo a alguien, o a alguien de alguien]. v Producir[le] angustia o congoja.

- registra los actantes por partida doble, en el lema y en la definición: no tener [uno] (ni) para empezar [con una pers. o cosa]. v (col) Ser [esa pers. o cosa] muy inferior a la capacidad o a la energía [de uno].

De lo anterior se desprende que el DFDEA no sigue criterios claros en su presentación de los elementos del contorno, o los sigue pero no los aplica de forma sistemática e igual en todas las unidades.

En este sentido, nos parece muy innovador y eficiente el procedimiento adoptado por Penadés Martínez, quien, en su DICLOCVER (2002) y los demás diccionarios que le siguieron, el DICLOCADV ${ }^{6}$ (2005) y el DICLOCNAP $P^{7}$ (2008), ha puesto de manifiesto que la sistematización de los elementos del contorno es un desafía posible de solventar. Como hemos comentado precedentemente, la autora aboga por incluir los actantes en un apartado independiente del lema. He aquí algunos ejemplos de locuciones presentadas de acuerdo con la concepción del DICLOCVER:

llevarse por delante v. [algo/alguien, algo/a alguien]

dar carpetazo v. [alguien, a algo].

dar vueltas la cabeza $v$. infor. [a alguien].

6 Diccionario de locuciones adverbiales para la enseñanza del español.

7 Diccionario de locuciones nominales, adjetivas y pronominales para la enseñanza del español. 
La misma autora (Penadés Martínez, 2015: 118-119) llama la atención sobre la necesidad de proporcionarle al usuario una serie de informaciones sobre los actantes, tales informaciones pueden referirse a:

— El número de los actantes: distingue aquí entre cuatro grupos de locuciones: las que no tienen ningún actante (avalentes): haber ropa tendida, un actante (monovalentes): hacerse el interesante (hacerse alguien el interesante), dos actantes (bivalentes): seguir las buellas (alguien sigue las buellas a 0 de alguien) y tres actantes (trivalentes): echar en cara (alguien echa algo en cara de alguien). Conviene señalar, en este sentido, que la estructura actancial de una locución determina su significado, esto es, un mismo fraseologismo puede tener varios significados si le corresponden varios esquemas argumentales, como se aprecia en los siguientes ejemplos (DFDEA):

quedarse colgado [alguien]. $v(c o l)$ Quedar defraudado de las expectativas que se le habían creado.

quedarse colgado [un ordenador o un programa]. $v$ (argot Informát) Bloquearse.

dar largas [a alguien]. $v$ Entretener[lo].

dar largas [a un asunto]. $v$ aplazar[lo].

Es de suma importancia señalar las distintas estructuras actanciales que pertenecen a la misma forma canónica. Solo así se puede delimitar el alcance de una locución con respecto a los actantes con que puede combinarse.

— la naturaleza semántica de los actantes: alguien, algo, algún lugar, una persona, una cosa, etc. El DFDEA, por ejemplo, suele informar con precisión sobre la naturaleza semántica de los argumentos, como en estos ejemplos:

echar (o poner) un remiendo [a una prenda].

poner precio a la cabeza [de una persona]

de porcelana [Cosa, esp. cutis]

no tener pierde [un lugar]

echarse a perder [una materia, esp. un alimento] 
— la función sintáctica de los actantes: en algunas entradas del DFDEA se hace mención de la función sintáctica de ciertas locuciones, aunque de modo irregular, a veces junto al lema, otras en la parte dedicada a la definición:

echar cuenta [a alguien (ci) o algo (ci o compl DE)].

habida cuenta. Teniendo presente o en consideración [algo (compl $D E+n$, $D E Q U E+v)]$.

\section{La inclusión del adverbio de negación "no"}

Además del régimen preposicional y los actantes, existen otros componentes que aparecen de forma irregular en el lema de los diccionarios consultados. Uno de estos elementos es el adverbio de negación no, el cual plantea el problema de si es o no un elemento constituyente de la forma canónica y, por ende, de decidir si incluirlo o descartarlo del lema de la locución. A continuación comentaremos distintos aspectos relativos al tratamiento de la partícula no en el lema.

En primer lugar se deja notar que los diccionarios tampoco están de acuerdo en una forma unificada para incluir el adverbio no en el lema. Obsérvense los siguientes ejemplos:

\begin{tabular}{|l|l|}
\hline \multicolumn{1}{|c|}{ DFEM } & \multicolumn{1}{|c|}{ DFDEA } \\
\hline 1. (no) venirle u.c. en gana a & \\
alguien & 1. venir en gana [algo a alguien] \\
2. (no) dar señales de vida & 2. dar señales de vida \\
3. (no) [estar u.p. ] en sus cabales & 3. en sus cabales \\
$\begin{array}{l}\text { 4. (no) irle ni venirle u.p./u.c. a co- } \\
\text { sa(suj)] alguien }\end{array}$ & 4. no irle ni venirle [a uno (ci) una \\
\hline
\end{tabular}

Tabla 2: La representación del adverbio de negación no en las locuciones recogidas en el DFEM y el DFDEA.

Son muchas las observaciones que se hacen tras la lectura de los ejemplos citados. Según se desprende del procedimiento seguido en el DFEM, este diccionario pone el adverbio de negación no entre paréntesis para indicar que la locución se puede usar en las dos formas, negativa y afirmativa. El caso del DFDEA es distinto, pues en los tres primeros ejemplos no incluye la partícula de negación, mientras que en la última locución sí aparece, ¿por qué? Este diccionario parece que incluye el no solo en los casos en que 
Ningún vecino abrió boca cuando la chica del tercero propuso echar al portero

Cuando la chica del tercero propuso echarlo, nadie abrió boca

Cuando la chica del tercero propuso echarlo, ni los vecinos ni el administrador abrieron boca

Los vecinos jamás abrieron boca para defenderlo

Entonces, ¿cuál será la forma más conveniente para registrar el adverbio no? De acuerdo con Penadés Martínez (cfr. 2015: 128) y según se deja entrever en el DFDEA es necesario incluir el no solo cuando la locución tiene un uso únicamente negativo y, por tanto, solo cuando forma realmente parte constitutiva del lema. En el caso de las locuciones que admiten las dos formas, se debe registrar únicamente la versión afirmativa, y dar cuenta del uso negativo en la definición o en los ejemplos, como hace el DFDEA con la locución dar señales de vida, en la que indica que se usa frecuentemente en oraciones negativas y lo confirma con dos ejemplos de uso:

- Los ratones no habían vuelto a dar señales de vida.

- Mientras escogía el bastón para el paseo, llamó a Mao. Pero el perro no dio señales de vida, y tampoco su sobrina.

Tomar una decisión con respecto a la inclusión o la eliminación de la partícula de negación no es, pues, una tarea fácil; por tanto, antes de decidir sobre esta cuestión, sería necesario realizar un estudio para examinar los distintos ejemplos de uso en contextos reales. Solo así se puede comprobar si el adverbio de negación constituye parte conexa de la locución o no.

\section{La forma pronominal "se"}

Al igual que los elementos tratados en los apartados anteriores, el registro del se en los diccionarios no se somete a un tratamiento unificado:

\begin{tabular}{|l|l|l|}
\hline \multicolumn{1}{|c|}{ DFDEA } & \multicolumn{1}{|c|}{ Clave } & \multicolumn{1}{c|}{ DLE } \\
\hline $\begin{array}{l}\text { ganarse la vida } \\
\text { comer (se) }(0 \\
\text { devorar) con los ojos } \\
\text { [a alguien o algo]. }\end{array}$ & $\begin{array}{l}\text { ganarse la vida } \\
\text { comer con los ojos a a a } \\
\text { alguien. }\end{array}$ & $\begin{array}{l}\text { ganar, o ganarse, } \\
\text { alguien la vida. } \\
\text { comerse alguien con } \\
\text { los ojos a otra } \\
\text { persona o algo. }\end{array}$ \\
\hline
\end{tabular}

Tabla 3: La representación de la forma pronominal se en las locuciones recogidas en el DFDEA, el Clave y el DLE. 
En el primer ejemplo (ganar la vida) tanto el DFDEA como El Clave han optado por presentar únicamente la forma pronominal (ganarse la vida), lo que da a entender que es la forma exclusiva en que se usa esta locución. Sin embargo, el DLE ha señalado los dos usos posibles (ganar, o ganarse), lo que significa que dicha unidad se puede usar en forma pronominal o no. La consulta del CREA ratifica que esta locución aparece mayormente en forma pronominal ( 254 casos en 214 documentos, efectuando la búsqueda solo con la forma pronominal "ganarse la vida", con "se ganaba la vida" se localizan 75 casos en 67 documentos). Los casos sin el se son muy pocos, por ejemplo realizando la búsqueda con "ganar la vida" hemos encontrado 18 casos en 17 documentos, de los cuales muchos aparecen también en forma pronominal como poderse ganar la vida, se tiene que ganar la vida, se quieren ganar la vida, me empecé a ganar la vida,... lo que hace que el número registrado de casos sin el se se reduzca aún más. He aquí algunos ejemplos:

Y no era un decir: era un impulso luminoso que vinculaba el ser y el parecer en una única intención: la intención de vivir en la verdad y de ganar la vida a pulso.

Hay el poeta de la Cibeles que va por el café tratando de ganar la vida vendiendo poemitas.

La falta de uniformidad entre los diccionarios citados plantea la duda de si considerar la partícula se como elemento opcional u obligatorio. No obstante, partiendo de los resultados obtenidos del CREA se puede constatar que son muy escasos los ejemplos en que la locución tratada aparece en la forma sin el se, dichos ejemplos no son tan representativos como para registrar la partícula se como elemento facultativo. Dicho esto, la locución ganarse la vida tiene que aparecer en el lema exclusivamente en la forma pronominal, al igual que otras locuciones que aparecen registradas únicamente con la partícula se, tales como abogarse en un vaso de agua, meterse en faena, dejarse los dientes, etc.

La unidad comerse con los ojos parece suscitar más polémica que el fraseologismo anterior, pues cada diccionario ha abogado por un procedimiento distinto a la hora de registrarla. De acuerdo con el DFDEA, el se es un elemento facultativo, porque lo pone entre paréntesis (comer (se)), para el DLE es obligatorio, porque solo recoge la forma pronominal (comerse) sin indicación de alternancia, mientras que el Clave lo descarta totalmente (comer), por lo que nos lleva a considerar que la locución en cuestión solo se usa en la forma sin el se. ¿Cuál de las tres formas es la más adecuada? Para contestar esta pregunta, tenemos que recurrir a los ejemplos de uso. El CREA registra muy pocos 
resultados de esta locución, sea en la forma con o sin el se, y ningún uso prevalece de modo considerable. He aquí algunos ejemplos:

La última vez que se habían visto, el mismo día del tiroteo ante el Eurobuilding, recordaba que había intentado llevársela al huerto y se la comía con los ojos.

Siguió el almuerzo, que sirvió Paulinha, ayudada del criado negro, que la comía con los ojos ante la indiferencia despectiva de la muchacha.

Respecto a los ejemplos que ofrecen los diccionarios, El DLE no brinda, en este sentido, ninguna ejemplificación. El Clave aunque no reconoce el uso de la forma pronominal en el lema, puesto que solo registra la forma sin el se, en el ejemplo de uso que ofrece deja patente el empleo de la forma con el se: "los dos enamorados se comían con los ojos". El DFDEA se muestra más coherente al presentar tres ejemplos, dos corresponden a ambos usos, y un tercero con el verbo devorar:

Resistes la cincuentena como pocos. Esta noche, Marisa Santoja, la condesa y, naturalmente, Rosa Fornell se te comían con los ojos.

Él la está comiendo con los ojos.

¿Qué salero tenía el dichoso Toniolo!...las mujeres lo devoraban con los ojos.

Los ejemplos de uso revelan que la locución tratada mantiene el mismo significado cuando se emplea con o sin el se ('Mirar[10] intensamente con muestras de deseo', DFDEA) y la misma combinatoria sintagmática (a alguien), por lo que el uso de esta partícula es opcional, y el lema debe plasmarlo mediante los paréntesis.

Si bien la locución anterior se puede usar indistintamente con o sin el se, porque la aparición o la ausencia de la partícula se no desencadena ningún cambio de significado ni de combinatoria, existe otra locución, comer con los ojos, que tiene otro significado: 'Apetecer o desear la comida cuando tiene un buen aspecto externo' (DLE). Tratándose de una locución polisémica, que puede activar dos significados distintos según el contexto en que esté insertada, esta debe figurar en dos entradas independientes, cada una con la definición y la ejemplificación correspondientes. He aquí otros ejemplos tomados del

DFDEA:

despertar a la vida [a alguien]. $v$ Hacer que vuelva a la vida tras un estado de anestesia, hibernación o muerte aparente. 
despertarse a la vida [alguien]. $v$ Comenzar a tener las primeras vivencias 0 impresiones de vida.

calentar la cabeza [a alguien]. $v$ (col) Cansar[le] o abrumar[le] con lo que se [le] dice.

calentarse [alguien] la cabeza. $v$ (col) Hacer un esfuerzo de reflexión.

Existe también otra clase de locuciones en las que la aparición del se no implica un cambio en el significado léxico, sino en el diatético (cfr. Penadés Martínez, 2002-2003: 113). Este tipo de locuciones ha de figurar también en dos entradas independientes, como en el caso de los siguientes ejemplos:

inclinar la balanza. $v$ Hacer que el asunto en cuestión se oriente [a favor o del lado de alguien o algo], DFDEA.

inclinarse la balanza. $v$ Orientarse el asunto en cuestión [a favor o del lado de alguien o algo], DFDEA.

El análisis de este tipo de unidades en los diccionarios revela la falta de criterios claros en su tratamiento. Si bien en el ejemplo anterior, el DFDEA ha presentado la locución inclinar la balanza en dos entradas distintas, para deslindar las diferencias diatéticas que se producen cuando la locución incluye el se, y cuando no, otros diccionarios han procedido de formas bastante dispares:

$D L E$ : inclinar la balanza.

Clave: inclinar la balanza.

$D S L E^{8}$ : inclinar (se) la balanza.

$L D P L^{9}$ : inclinar o inclinarse la balanza.

DFEM: inclinar/inclinarse la balanza.

Se puede decir que los ejemplos citados reflejan un problema de carácter práctico relacionado con el espacio del diccionario, y es precisamente lo que explica por qué todos los repertorios antes mencionados incluyen la locución inclinar la balanza en una sola entrada, aunque lo hacen de distintas maneras: mientras el $D L E$ y el Clave no muestran ninguna indicación de alternancia, los demás repertorios lo hacen de diferentes modos:

8 Diccionario Salamanca de la lengua española.

9 Larrousse diccionario práctico de locuciones. 
el DSLE mediante los paréntesis redondos, el $L D P L$ con la conjunción o, y el DFEM mediante una barra. De ahí, se desprende que la ausencia de criterios en el tratamiento del se es notable, no solo en el hecho de incluir la locución en una o dos entradas, sino también en cómo indicar la alternancia.

Ciñéndonos a los resultados de la descripción realizada en este apartado, podemos establecer los casos en que debe registrar el se en el lema:

- $\quad$ se registrará entre paréntesis solo cuando es un elemento facultativo (comer(se) con los ojos).

- $\quad$ se registrará con el se, sin indicación de alternancia, solo cuando se usa exclusivamente en la forma pronominal.

- $\quad$ se registrará sin el se solo cuando se usa únicamente en la forma no pronominal.

- $\quad$ se registrará en dos entradas distintas cuando tiene dos significados distintos: comer con los ojos vs comer (se) con los ojos.

- $\quad$ se registrará en dos entradas distintas cuando tiene diferencias en el significado diatético: inclinar la balanza vs inclinarse la balanza.

\section{Conclusiones}

En definitiva, tomar una decisión con respecto a la inserción o no de cualquier elemento en el lema de una locución, sea el adverbio de negación no, los elementos del contorno, la partícula se o la preposición de, depende, como ya hemos dicho en muchas ocasiones, de los ejemplos de uso. Son muy importantes, en este aspecto, los estudios de fraseología teórica que se basan en los ejemplos de uso para analizar el funcionamiento discursivo de las UFS, dar cuenta de la frecuencia de uso de cada elemento dentro de la unidad a la que pertenece y tratar ciertos aspectos como el significado diatético. Contando con estas investigaciones, los fraseógrafos podrán delimitar, con más exactitud, la(s) forma(s) que deben adoptar las locuciones en el lema. Pensamos, en este sentido, que la determinación del lema depende, en gran medida, del provecho que se saque de los logros de la fraseología teórica, por lo que el establecimiento de la forma canónica de una locución debe llevarse a cabo con posterioridad a la elaboración de estudios fraseológicos centrados en ejemplos de uso. Lo dicho muestra, una vez más, la interdependencia entre la fraseología y la fraseografía. 


\section{Referencias}

Bobes Soler, E. (2016). Información gramatical asociada a las locuciones verbales del español. Tesis Doctoral. Salamanca: Universidad de Salamanca.

Castillo Carballo, M. (2015). De la investigación fraseológica a las decisiones fraseográficas: un estudio de interrelaciones. Vigo: Academia del Hispanismo.

Conca I Martínez, M. (1998). «0s estudios de fraseoloxía catalana: realidades e proxectos». En FERRO RUIBAL, Xesús (ed.). Actas do I Coloquio Galego de Fraseoloxía. Vigo: Xunta de Galicia, 139-167.

Martínez Marín, J. (1996). Estudios de fraseología española. Málaga: Ágora.

Mellado Blanco, C. (2004). Fraseologismos somáticos del alemán. Frankfurt am Main: Peter Lang.

Mellado Blanco, C. (2017). «El contorno lexicográfico en Fraseología: naturaleza y ubicación en la microestructura de los diccionarios generales y fraseológicos». En MOGORRÓN HUERTA, Pedro; ALBALADEJO-MARTÍNEZ, Juan Antonio (eds.). Fraseología, diatopía y traducción. Phraseology, Diatopic Variation and Translation (colección IVITRA Research in Linguistic and Literature). Amsterdam: John Benjamins , 107-136.

Montoro Del Arco, E. (2005). Hacia una sistematización de la variabilidad fraseológica. En PASTOR MILÁN, Ma Ángeles (ed.). Estudios lingüísticos en recuerdo del profesor Juan Martínez Marín. Granada: Editorial Universidad de Granada, 125-152.

Olímpio De Oliveira Silva, M. (2004). Fraseografía teórica y práctica. Bases para la elaboración de un diccionario de locuciones verbales español-portugués. Tesis doctoral. Alcalá de Henares: Universidad de Alcalá.

Penadés Martínez, I. (2002-2003). «La elaboración del Diccionario de locuciones verbales para la enseñanza del español (DICLOCVER)». Revista de Lexicografía, IX, 97-129.

Penadés Martínez, I. (2008). Proyecto para la redacción de un diccionario de locuciones del español. En Bernal, Elisenda; DECESARIS, Janet (eds.). Proceedings of the XIII EURALEX International Congress. Barcelona, IULA: Universitat Pompeu Fabra, 1379-1384. 
Penadés Martínez, I. (2010). El Diccionario de locuciones del español actual (DILEA). En MELLADO BLANCO, Carmen et al. (eds.). La fraseografía del S. XXI. Nuevas propuestas para el español y el alemán. Berlín: Frank \& Timme, 191-210.

Penadés Martínez, I. (2015). Para un diccionario de locuciones, de la lingüística teórica a la fraseografía práctica. Universidad de Alcalá: Servicio de Publicaciones.

Santamaría Pérez, M. (2001). Tratamiento de las unidades fraseológicas en la lexicografía bilingüe español-catalán. Alicante: Biblioteca virtual Miguel de Cervantes.

Serra Sepúlveda, S. (2006). Gramática y diccionario. El problema del contorno en lexicografía española. Boletín de Filología, Tomo XLI, 197-240.

Torrent-Lenzen, A. (2007). El Diccionario español-alemán de fraseologismos idiomáticos de Colonia: un proyecto en curso. Estudis Romànics XXIX: 279-289.

Werner, R. (1982). La unidad léxica y el lema. En HAENSCH, Günther et al. (eds.). La lexicografía. De la lingüística teórica a la lexicografía práctica. Madrid: Gredos, 188-232.

Zuluaga, A. (1992). Spanisch: Phaseologie. Fraseología. En Günter Holtus et al. (eds.).

Lexikon der Romanistischen Linguistik, VI/1. Tübingen, Niemeyer, 125-131.

\section{Diccionarios}

Clave: Maldonado, C. (dir.) (1996). Clave. Diccionario de uso del español actual. Madrid:

S.M. http://clave.smdiccionarios.com/app.php

DFDEA: Seco, M. (dir.) et al. (2004). Diccionario fraseológico documentado del español

actual. Locuciones y modismos españoles. Madrid: Aguilar.

DFEM: Varela, F.; Kubarth, H. (1994). Diccionario fraseológico del español moderno.

Madrid: Gredos.DICLOCADV: Penadés Martínez, I. (2005). Diccionario de locuciones adverbiales para

la enseñanza del español. Madrid: Arco/Libros.

DICLOCNAP: Penadés Martínez, I. (2008). Diccionario de locuciones nominales, adjetivas y pronominales para la enseñanza del español. Madrid: Arco/Libros. 
DICLOCVER: Penadés Martínez, I. (2002). Diccionario de locuciones verbales para la enseñanza del español. Madrid: Arco/Libros.

DLE: Real Academia Española (2014). Diccionario de la Lengua Española. Madrid: Espasa. Versión en línea: http://DLE .rae.es/

DSLE: Gutiérrez Cuadrado, J. (dir.) (1996). Diccionario Salamanca de la lengua española. Madrid: Santularia/ Universidad de Salamanca.

LDPL: Fontanillo Merino, E. (dir.) (1993). Larousse diccionario práctico de locuciones. Barcelona: Larousse Planeta. 